\title{
Comparison of traditional lecture and flipped classroom for teaching programming
}

\section{Dieter Pawelczak}

Faculty of Electrical Engineering and Computer Science, Universitaet der Bundeswehr Muenchen, Neubiberg, Germany

\begin{abstract}
Programming courses in undergraduate education seem to be predestined for a flipped classroom approach as learning programming requires a high personal contribution on the one hand and on the other hand, course participants typically start with a wide range of previous knowledge and skills. Within a flipped classroom students can organize their learning phases self-reliantly and put an individual amount of effort into each learning objective. Whilst in a traditional lecture it is not easy to motivate students, the flipped classroom requires students' active involvement per se. Besides all these advantages, setting up such a course requires a high initial effort for the lecturer. Furthermore, students might prefer a lecture, as usually the work load is higher in a comparable flipped classroom course.

Based on the idea of flipping a beginners programming course, we firstly explored the effects of a flipped classroom approach on an elective advanced programming course with a smaller student group. The paper compares the new course design and its effects on the students learning, on the teaching, as well as on the course preparation with the former traditional lecture. The comparison is based on a survey, the students' evaluation feedback and on the examination results.
\end{abstract}

Keywords: flipped classroom; computer science education; teaching programming. 


\section{Introduction}

Students enrolled in engineering often have difficulties with learning a programming language. The reasons are wide-spread. Butler and Morgan (2007) identify in a extensive survey among novice programmers in particular problems with abstract principles and logic. Bosse and Gerosa (2017) give an overview on the literature about programming difficulties. There are many different measures to enhance students' learning; starting from special programming languages to interactive visualization of programming concepts. We use, e.g. the Virtual-C IDE as a programming environment, which is especially designed for beginners, see Pawelczak and Baumann (2014). But this paper focuses more on teaching methods than on tools. Despite difficulties with abstract concepts Lahtinen et al. (2005) also find in their study, that most helpful for students' learning is writing programs on their own.

So, learning programming requires a high personal contribution. On the other hand, students' previous knowledge typically varies: some might have none or only limited experience with programming languages, some are already skilled or have developed misconceptions. For both scenarios, the concept of a flipped classroom (FC) appears promising, as it strengthens the self-engagement of the students: they have to prepare themselves on the basis of adequate course material; regular tests on the learning objectives and programming assignments keep students busy with the study matters and a review session gives the opportunity to detect misconceptions early. Although our primary focus is the education of novice programmers, we first want to test the FC on a smaller student group. We therefore use an elective advanced programming course. This paper presents the new course design and compares it with the former traditional lecture (TL) with respect to the students learning, to the teaching, as well as to the course preparation. We run a survey at the end of each course and examined both, the students' evaluation feedback and the examination results.

\section{Related Work}

FC is a common student-centered active learning method and has a rising prevalence in undergraduate education, compare Giannakos et al. (2014). Some benefits compared to a TL are the positive attitudes, and the increased engagement of the students. Although most studies emphasize an increased learning performance, there is no common agreement, that FC reveals better or more sustainable learning results. Kaplan (2015) e.g. compares a traditional CS1 course with an FC and describes typical challenges and draws the conclusion that FC students require more time to learn the same content. He also describes, that the effort for developing such a course is significantly higher. Lepp and Tonisson (2015) analyze the successful implementation of a beginners' programming course at Tartu University with about $70 \%$ of the students thinking that they learned better and more compared to a TL. With regards to the high effort required to establish such a course, Lepp and Tonisson benefit from automated videotaping of lectures and can therefore refer to a large pool of 
video material. Although not generally required for flipping a course, the use of videos is wide-spread and often discussed as a major benefit, which results in a better acceptance by students. Still, watching videos is time consuming and adds another common drawback of an FC: in TL students are trained independently of their preparation, while in FC students have to prepare themselves. Dazo et al. (2016) therefore analyze the video viewing behavior of course participants. They notice, that the viewing rate drops immediately after course begin and rises again before the examination. As course preparation is crucial, they suggest an automated reminder for students, to watch the videos prior to the lecture. Falling course attendance and students struggling with self-organization are other facts, that arise with the FC approach. For this reason, Köppe et al. (2015) published some guidelines on the in-class meetings to overcome these difficulties.

From the literature review, the objective of our research is to explore the effort for both lecturer and students - for a course based on videos, clarify the acceptance of videos and get a sense, if flipping the beginners' programming course is feasible as well. We keep in mind, that despite the success Lepp and Tonisson (2015) described, they performed the FC only with smaller student groups and used TL for large enrollments.

\section{Former and New Course Setup}

The course is an advanced $\mathrm{C} / \mathrm{C}++$-programming course. It is designed to expand a previous C course for beginners.

\subsection{Former Course Setup}

The former course comprised two hours per week each for lecture and lab work with seven programming assignments. In the first weeks, there is lecture only, while in the last seven weeks the lecture accompanies the lab work; typically, the lecture contents are 1-2 weeks ahead of the lab. The lecture prepares students for the lab work, but is kept quite general to give a wide overview on different topics. At the end of the course, there is a 2 hours final lecture and a 90 minutes written examination. The overall presence time is 4 hours a week. The preparation time is expected to be up to 3.5 hours, depending on the student's skills. The course material consists of a detailed script, a list of book references, lecture slides and the work sheets for the lab work.

\subsection{The New Course Setup}

The new course concept starts with a 30-minutes kick-off, which gives a short introduction on the course contents, but mainly describes the course setup. The focus of the course is now the lab work: each 2-hours lab requires additional course material compared to the TL:

- Preparation guide: a short document describing the subjects of the next lab work: chapters to be read in the script, videos to be viewed and further reading information 
in the script/ in books/ or in online sources. The guide also provides a set of questions to test the learning objectives.

- Video material: video cuts from the former courses lectures: the video material of 2 hours lecture is typically shortened to 1 or 2 videos with 10-30 minutes lengths.

- Online questionnaire: a quiz consisting of various questions from the preparation guide plus some small transfer tasks. In addition, it allows students to ask their own questions. The quiz has no direct feedback - it just collects questions and answers.

The time schedule for the lab work is organized as follows:

- A week in advance: release of the preparation guide \& video material.

- Three days before the lab work:

○ issuing the work sheet for the lab work,

- activating the online quiz,

o invitation of the students to participate in the quiz.

- Review session the day before the lab: the lecturer moderates a 30-45 minutes discussion with the students about the questions and answers.

- After the lab: students get the next preparation guide and the schedule repeats.

The course ends like the former course with a final lecture and the written examination. The overall presence time is 2.5 - 3 hours a week. As a minimum, an hour per week is expected for video-based learning and answering the online quizzes. We estimated approximately two hours additional preparation time for the lab and for working with script and sources.

\subsection{The Transition to the New Course Setup}

The lecture of the TL course was videotaped in 2015. Students of this course already got access to the video material. The videos were typically put online a week after the lecture. The deployment of the videos had a negative effect on the lecture attendance: whilst in former lectures the attendance rate was typically about $80 \%$, it now dropped down to $64 \%$.

\section{Data and Methodology}

\subsection{Course Evaluation}

For the comparison of the courses a regular student evaluation is performed for each course with additional specific questions on the course material (both courses) and on the FC (only new course). The survey uses the Likert scale.

\subsection{Data Basis and Comparison Setting}

The survey was performed online in both courses, each time in the week before the last lab work. From 19 students in 2015, only 10 answered the survey, in 2016 we received the feedback from 10 out of 12 students. In addition to the survey outcome, we used the results 
from the examinations. As students have no access to earlier examinations, we used exactly the same examination for a better comparison with some lexical modifications only. These were also marked according to the same scheme. For the lab work, the same work sheets were applied. In order to get an unbiased comparison, the course description was not modified: students learned in the first lesson that the course modalities had changed.

\section{Results}

\subsection{Survey Results for the Flipped Classroom}

As discussed in section 2, we expected a higher effort for course participants compared to the traditional lecture. As figure 1 shows, the majority of the participants disagreed on a higher effort (Q1 \& Q2). One student stated the overall effort as higher. Of course, the results emphasize our intention of shaping the course with similar work-load. On the other hand, the overall learning content is reduced due to the focus on the lab work and less on background knowledge. Some video material from the traditional lecture was not issued to the students as it provides additional information, but is not directly related to the lab work.

Q1: The effort for the course preparation is much higher compared to a traditional lecture.
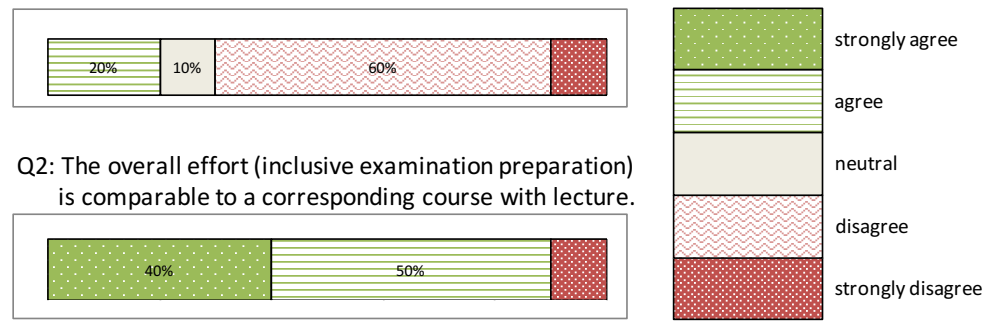

Figure 1. Q1 \& Q2: Survey results with respect to the effort of the flipped classroom approach

Students were very content with the new course: $70 \%$ stated that the course was more fun compared to a lecture-based course. Even $90 \%$ agreed that they feel better prepared by themselves, compared to attending an equivalent lecture, compare Q4 in figure 2. This is the first course with the flipped classroom approach at our faculty; therefore, the new concept is certainly more approved. From the written comments in the survey, it was suggested to videotape and publish the review sessions as well. Of course, this would give students more freedom in their time to learn, on the other hand it contradicts the fact that some students need to be pushed to do their preparation: the average attendance rate of the review session was with $57 \%$ lower than the attendance rate of the traditional lecture (64\%). The download rate of videos usually resembled the number of participants in the review session, but rose again before the lab work and the exam. We might conclude, that students who had no time for preparation, did not come to the review sessions. 
Q3: The course makes more fun compared to an equivalent lecture based course.

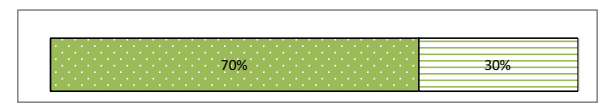

Q4: I feel better prepared due to my own work on the course material compared to attending an equivalent lecture

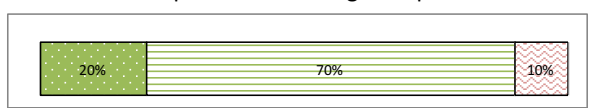

Figure 2. Q3 \& Q4: Survey results regarding course enjoyment and self-assessment related to learning progress

\subsection{Comparison to the Traditional Lecture}

The standard course evaluation showed only tiny differences. On a German grading scheme (1- very good to 5- poor quality) the TL was rated 1.13 while FC received 1.17 . Due to the small quantity of data, the difference is negligible, but as other results reveal, one student in the FC group obviously preferred the traditional lecture.

Q5: I would also use raw (unedited) videos for learning

\begin{tabular}{|l|l|l|l|l|l|l|}
\hline $22,2 \%$ & $33,3 \%$ & $30 \%$ & $20 \%$ & $30 \%$ \\
\hline \hline
\end{tabular}

a) traditional lecture

b) flipped classroom

Q6: Would I wish more detailed videos?

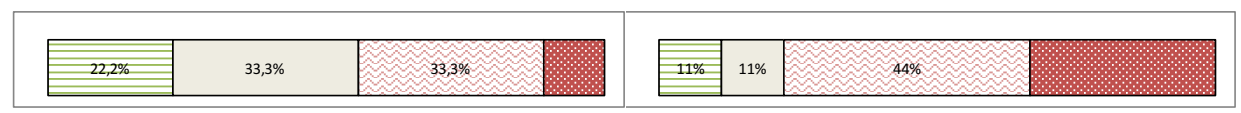

a) traditional lecture

b) flipped classroom

Q7: Videos can supplement a lecture but cannot replace it?

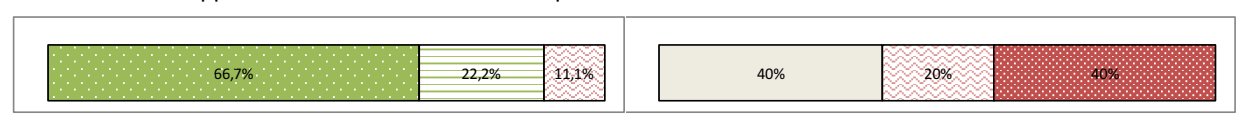

a) traditional lecture

b) flipped classroom

Figure 3. Q5 - Q7: Preferences regarding the video material in comparison.

As the effort for creating short and concise videos is very high, we asked the question if students would also use unedited video material from the lecture, compare figure 3 . Interestingly more students from FC agreed on that, in TL more students declined that (Q5). Even more students disagreed on more detailed video material in FC, which indicates, that the effort of watching the videos is already high (Q6). We also asked the provocative question, whether videos can support a lecture but not replace it (Q7). In TL $90 \%$ agreed with that, while in FC $60 \%$ disagreed with that. 


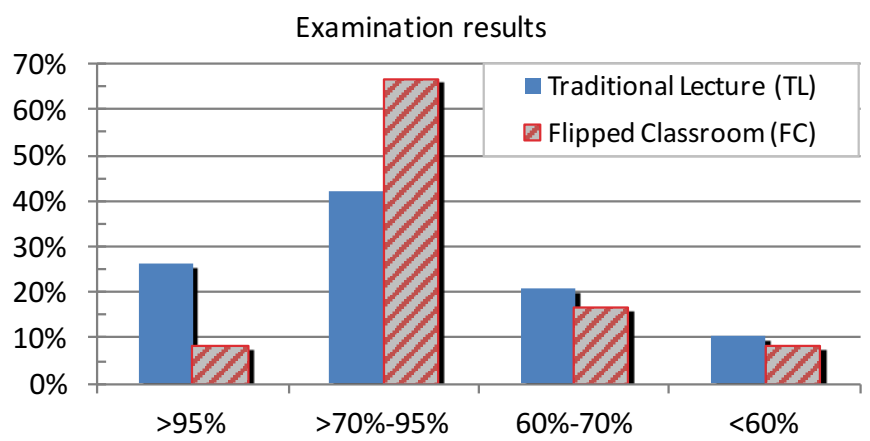

Figure 4. The examination results of traditional lecture and flipped classroom in comparison. The average points reached were 79.5 out of 100 for TL and slightly less with 78.1 points for FC.

The results from the examinations are hard to compare, as the group sizes are very small. The average points reached were quite similar, but the TL received slightly better results, compare figure 4: there is a higher number of best grades in the TL and the grades are more widely spread. It might imply, that TL clarified more details, or, that a misconception arose in the self-learning phase and the review session could not straighten it out.

\subsection{Lecture's Experience with Flipped Classroom}

The advantage compared to the TL is the strong focus in the review session: while TL often requires extra explanation and the lecturer has to go back further, the review session can concentrate on details. Of course, not all students are well prepared, still the level of detail is distinctly higher compared to the TL. This also has an impact on the learning atmosphere: the review session becomes an active discussion compared to a more unidirectional lecture and is "more fun" as the students stated.

The effort to set up the new course was very high. In future, adaption of the course material will be required and will again demand a high investment in time. Although the presence time of the lecturer was reduced by 1 to 1.5 hours per week, the overall time effort is definitive less for a TL.

\section{Conclusion and Outlook}

The benefits of the new course design are the high acceptance by the students as they seem more motivated in working with the course material at arbitrary times and - as stated in the survey - enjoyed the flipped classroom approach very much. The examination results show only tiny differences compared to previous courses and the students' workload was not reported as higher. The lecturer experienced a more inspiring learning environment as students were better prepared and discussions could be established on a higher level. We will therefore keep the flipped classroom concept for the course in the future. Besides the advantages, the flipped classroom course has drawbacks, i.e. the overall learning content is 
reduced, as the focus is more on the lab work than on theory. We therefore plan optional additional tasks in the lab to extend the learning objects for interested students without increasing the general workload. The effort of setting it up was very high and as programming languages are evolving fast, the course material needs to be updated continuously. Despite the students' motivation, a tendency is discernible, that students delay their course preparation or skip the review session as they do not manage to prepare in time. It is hard to transfer the experiences to a beginners' course, as it is an elective course, which students choose, that feel familiar with programming. Probably more changes need to be made to motivate programming novices. Therefore, we refrain from our initial intention to flip the beginners' course for now as we want to gain more experience, first.

\section{References}

Bosse, Y., \& Gerosa, M. A. (2017). Why is programming so difficult to learn? Patterns of Difficulties Related to Programming Learning Mid-Stage. SIGSOFT Softw. Eng. Notes 41, 6 (January 2017), 1-6

Butler, M., \& Morgan, M. (2007). Learning challenges faced by novice programming students studying high level and low feedback concepts. In ICT: Providing choices for learners and learning. Proceedings ascilate Singapore 2007, 99-107

Dazo, S. L., Stepanek, N. R., Fulkerson, R., \& Dorn, B. (2016). An Empirical Analysis of Video Viewing Behaviors in Flipped CS1 Courses. In Proc. of the 2016 ACM Conf. on Innovation and Technol. in Comput. Sci. Educ. (ITiCSE '16). New York, USA, 106-111

Giannakos, M. N., Krogstie, J., \& Chrisochoides, N. (2014). Reviewing the flipped classroom research: reflections for computer science education. In Proc. of the Comput. Sci. Educ. Research Conf. (CSERC '14). ACM, New York, NY, USA, 23-29

Kaplan, R. M. (2015). Using Problem-Based Learning in a CS1 Course -Tales from the Trenches-. In Proc. of the Intern. Conf. on Frontiers in Educ.: CS and CE (FECS'15), Las Vegas, Nevada, USA, July 27-30, 2015, 86-90.

Köppe, C., Niels, R., Holwerda, R., Tijsma, L., Van Diepen, N., Van Turnhout, K., \& Bakker, R. (2015). Flipped classroom patterns: designing valuable in-class meetings. In Proc. of the $20^{\text {th }}$ European Conf. on Pattern Languages of Programs (EuroPLoP '15). ACM, New York, NY, USA, Article 26

Lahtinen, E., Mutka, K. A., \& Jarvinen, H. M. (2005). A Study of the difficulties of novice programmers, In Proc. of the $10^{\text {th }}$ Annual SIGCSE Conf. on Innovation and Technol. in Comput. Sci. Educ. ITiCSE'05, 14-18

Lepp, M., \& Tonisson, E. (2015). Integrating Flipped Classroom Approach and Work in Pairs into Workshops in Programming Course. In Proc. of the Intern. Conf. on Frontiers in Educ.: CS and CE (FECS'15), Las Vegas, Nevada, USA, July 27-30, 2015, 220-226

Pawelczak, D. \& Baumann, A. (2014): Virtual-C - a programming environment for teaching $\mathrm{C}$ in undergraduate programming courses. In Proc. of the IEEE Global Engineering Educ. Conf. (EDUCON), 3-5 April 2014, Istanbul, Turkey, 1142-1148 